CVIA

CASE REPORT

pISSN 2508-707X / elSSN 2508-7088 https://doi.org/10.22468/cvia.2018.00087 CVIA 2018;2(3):150-151

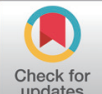

Received: April 2, 2018

Revised: May 5, 2018

Accepted: May 29, 2018

Corresponding author

Yavuzer Koza, MD

Department of Cardiology,

Ataturk University Faculty of Medicine,

Yakutiye, Erzurum 25100, Turkey

Tel: 90-442-2318521

Fax: 90-442-2361301

E-mail: yavuzerkoza@hotmail.com

\section{An Elderly Man with Heart Failure and Groin Pain: The Importance of the Psoas Sign and Advanced Imaging}

\author{
Yavuzer Koza, Hakan Taş, Mustafa Özkoç \\ Department of Cardiology, Ataturk University Faculty of Medicine, Erzurum, Turkey
}

\begin{abstract}
lliopsoas hematoma (IPH) is a rare and potentially life-threatening illness. The clinical symptoms can be non-specific hip, groin, or back pain with no external evidence of mass on examination and can be overlooked as musculoskeletal pain. Early diagnosis is important because early treatment results in better recovery and fewer complications. Contrast-enhanced computed tomography is the most sensitive method for confirmation of hematoma. We are reporting a case of IPH accompanied by severe hemorrhagic shock.
\end{abstract}

Key words Heart failure - Hematoma - Anticoagulants - Musculoskeletal pain.

\section{INTRODUCTION}

Iliopsoas hematoma (IPH) is a rare but potentially serious complication that can occur in anticoagulated patients. The symptoms and natural history range from slight pain to femoral neuropathy or precipitous shock and cardiovascular collapse [1]. We report an unusual case of spontaneous IPH accompanied by severe hemorrhagic shock.

\section{CASE REPORT}

An 85-year-old male patient was hospitalized with the diagnosis of decompensated chronic heart failure. His past medical history included coronary artery disease, diabetes mellitus, prostate cancer, and atrial fibrillation. On physical examination, he had positive pretibial edema $(+3)$ and rough breath sounds. His heart rate was 120 beats per minute, and blood pressure was 110/70 $\mathrm{mm} \mathrm{Hg}$. His home medications were monopril, aspirin, repaglinide, carvedilol, atorvastatin, and furosemide. He had no personal history of trauma or bleeding event for several months prior to admission. Echocardiogram revealed a left ventricular ejection fraction of $30 \%$ with global hypokinesia and pulmonary hypertension. Enoxaparine $(0.3 \mathrm{mg} / \mathrm{kg}$ once daily) was started for deep vein thrombosis prophylaxis and

(c) This is an Open Access article distributed under the terms of the Creative Commons Attribution Non-Commercial License (https://creativecommons.org/licenses/bync/4.0) which permits unrestricted non-commercial use, distribution, and reproduction in any medium, provided the original work is properly cited. atrial fibrillation because he had a low body weight ( $57 \mathrm{~kg}$ ), and the creatinine clearance was less than $30 \mathrm{~mL} / \mathrm{min}$. The platelet cell count, prothrombin time, and activated partial thromboplastin time were within normal ranges. On the fifth day of admission, the patient reported right groin and hip pain with restriction of right hip movement. On physical examination, there was extreme sensitivity in the right groin and hip with flexion and extension, known as the psoas sign. Abdominal ultrasonography showed loculated fluid in the psoas muscle. Abdominal computed tomography (CT) revealed a right iliopsoas muscle hematoma (Fig. 1). The general surgery and orthopedic clinics were consulted for urgent surgery. Despite packed red blood cell concentrate and fluid transfusions, the control hemoglobin level dropped from $11 \mathrm{gr} / \mathrm{dL}$ to $6 \mathrm{gr} / \mathrm{dL}$. After a few hours, the patient's blood pressure dropped, and cardiovascular collapse developed. Cardiopulmonary resuscitation was started, but the patient died. Informed consent was obtained.

\section{DISCUSSION}

IPH is defined as a spontaneous or traumatic retroperitoneal collection of blood involving the iliopsoas muscle. The incidence of retroperitoneal bleeding in patients undergoing anticoagulation has been reported to range from $0.1 \%$ to $0.6 \%$ [2]. Risk factors for spontaneous IPH include bleeding diathesis, trauma, anticoagulant treatment, and advanced age. Although the pathogenesis and pathophysiology of retroperitoneal bleed- 

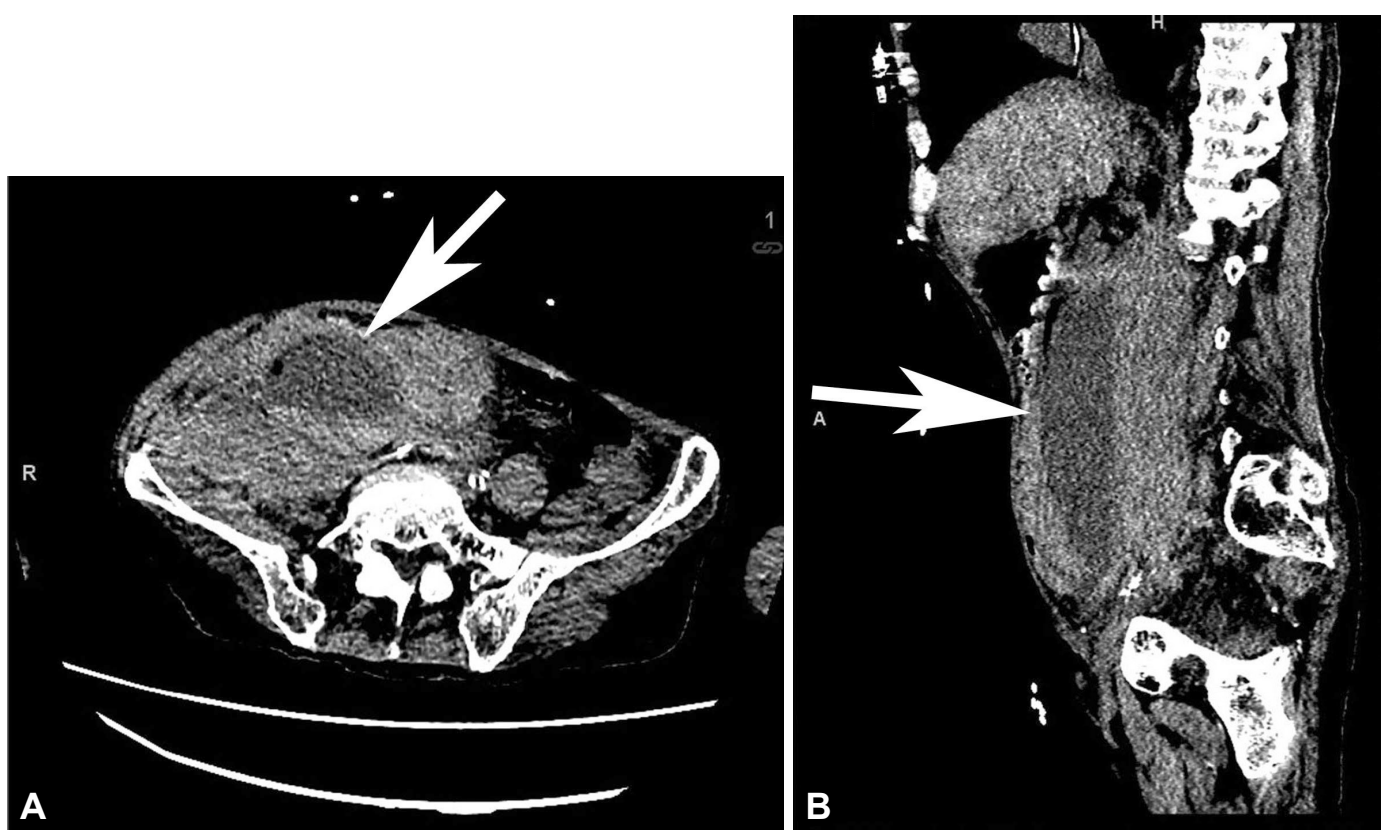

Fig. 1. Computed tomography of the abdomen: axial (A) and sagittal (B) views showing right iliopsoas hematoma (arrows).

ing remain unknown, retroperitoneal microvascular atherosclerosis, anticoagulation, and microtrauma, such as cough or vomiting, could lead to increased sensitivity to rupture [3].

The clinical presentation depends on the severity of the hemorrhage, and presentation can vary from compressive symptoms, such as nerve palsy or ischemia, to lower abdominal pain and/or anemia. A large amount of bleeding may cause hypovolemic shock and abdominal compartment syndrome, as in our case. In a multicenter study of patients with a diagnosis of spontaneous IPH in intensive care, hemorrhagic shock was the most common clinical presentation after compressive symptoms, and a high percentage of patients were diagnosed incidentally. Interestingly, there was not an association between anticoagulant therapy type and outcome [3].

Our patient reported right groin and hip pain, and this was initially thought to be due to arthritis. IPH can be misdiagnosed as musculoskeletal pain in the absence of a mass or bruise on examination. Moreover, the chance of missing an IPH is high in this clinical presentation because pain that is exacerbated with movement of the hip in an elderly patient usually suggests a musculoskeletal disorder rather than active bleeding.

Contrast-enhanced CT is the most sensitive method for diagnosis, providing useful information on volume, spatial extent, compressive complications, and/or active bleeding. Magnetic resonance imaging is more sensitive and specific, but its use is restricted as it is time consuming, expensive, and cannot be used in patients with metallic implants $[3,4]$.
Treatment of IPH depends on the speed of hemorrhage, hemodynamic status, and neurological deficit. Small hematomas usually require conservative management. Surgical intervention is mandatory for severe motor dysfunction or hemorrhagic shock [5].

In conclusion, IPH is troublesome for both clinicians and surgeons. The risk of hemorrhage is not associated with the amount of heparin administered, and hemorrhage can occur even at therapeutic levels. Physicians should include IPH in differential diagnosis, before musculoskeletal pain, when treating a patient on anticoagulant therapy with new onset or worsening hip and groin pain.

\section{Conflicts of Interest}

The authors declare that they have no conflict of interest.

\section{REFERENCES}

1. Butt MU, Buzsaki LA, Smyth SS, Elayi SC. Deep vein thrombosis complicated by spontaneous iliopsoas hematoma in patient with septic shock. Am J Case Rep 2017;18:1148-1152.

2. Estivill Pallejà X, Domingo P, Fontcuberta J, Félez J. Spontaneous retroperitoneal hemorrhage during oral anticoagulant therapy. Arch Intern Med 1985;145:1531-1534.

3. Llitjos JF, Daviaud F, Grimaldi D, Legriel S, Georges JL, Guerot E, et al. Ilio-psoas hematoma in the intensive care unit: a multicentric study. Ann Intensive Care 2016;6:8

4. Zago G, Appel-da-Silva MC, Danzmann LC. Iliopsoas muscle hematoma during treatment with warfarin. Arq Bras Cardiol 2010;94:e1-e3.

5. Kim SW, Kim DH, Jung SN. Spontaneous iliopsoas hematoma: a rare complication of anticoagulant use. Arch Plast Surg 2015;42:507-510. 